\title{
Quantitative analysis of multiphase magnetic resonance images may assist prediction of histopathological grade of small hepatocellular carcinoma
}

\author{
Shuping Weng', Xuru Xü ${ }^{2,3}$, Yueming $\mathrm{Li}^{3,4}$, Chuan Yan ${ }^{3}$, Jianwei Chen ${ }^{3}$, Rongping Ye ${ }^{3}$, Yuemin Zhu ${ }^{3}$, \\ Liting Wen ${ }^{3}$, Jinsheng Hong ${ }^{4,5}$ \\ ${ }^{1}$ Department of Radiology, Fujian Maternity and Child Health Hospital, Fuzhou, China; ${ }^{2}$ Department of Radiology, Zhongshan Hospital Affiliated \\ to Xiamen University, Xiamen, China; ${ }^{3}$ Department of Radiology, the First Affiliated Hospital of Fujian Medical University, Fuzhou, China; ${ }^{4}$ Key \\ Laboratory of Radiation Biology (Fujian Medical University), Fujian Province University, Fuzhou, China; ${ }^{5}$ Department of Radiation Oncology, the \\ First Affiliated Hospital of Fujian Medical University, Fuzhou, China \\ Contributions: (I) Conception and design: S Weng, Y Li; (II) Administrative support: Y Li, S Weng; (III) Provision of study materials or patients: \\ S Weng, X Xu, C Yan; (IV) Collection and assembly of data: S Weng, X Xu, J Chen, R Ye, Y Zhu, L Wen; (V) Data analysis and interpretation: S \\ Weng, X Xu, Y Li, J Chen, C Yan, J Hong; (VI) Manuscript writing: All authors; (VII) Final approval of manuscript: All authors. \\ Correspondence to: Yueming Li, MD. Department of Radiology, the First Affiliated Hospital of Fujian Medical University, Fuzhou, China. \\ Email: fjmulym@163.com.
}

Background: The aim of the study was to investigate whether preoperative quantitative analysis of multiphase magnetic resonance images may assist in predicting the pathological grade of small hepatocellular carcinoma (HCC).

Methods: A total of 49 patients with small HCCs $(\leq 3 \mathrm{~cm})$ underwent multiphase magnetic resonance imaging (MRI) and were retrospectively reviewed. Routine unenhanced and post gadobenate dimeglumine (Gd-BOPTA)-enhanced MRI were preoperatively performed. Signal intensity (SI) was measured within the designated region of interest (ROI) including those of the lesion and paraspinous muscles. The lesion-toparaspinous muscle relative contrast ratio (RCR) on T2-weighted (T2W) imaging, diffusion-weighted (DW) imaging, and dynamic phase Gd-BOPTA-enhanced T1W (T1-weighted) imaging were calculated, and statistical analysis was performed to determine the predictive power for the histological grade.

Results: In all, 49 cases were included comprising 3 well-differentiated (WD) HCCs, 36 moderately differentiated (MD) HCCs, and 10 poorly differentiated (PD) HCCs. There was a negative correlation between the RCR and pathological grade of small HCC in the arterial phase [correlation coefficient $(\rho)=-0.305, \mathrm{P}<0.05]$. However, there was no correlation between RCR in other phases and pathological grade $(\mathrm{P}>0.05$ for all). There was also no correlation between tumor margin, tumor location, cystic/necrotic change, intratumoral fat, enhancement pattern, tumor capsule, tumor boundary or tumor size, and any of the differentiation categories $(\mathrm{P}>0.05$ for all).

Conclusions: The lesion-to-paraspinous muscle RCR on arterial phase Gd-BOPTA-enhanced T1W imaging may be useful for the prediction of the histological characteristics of small HCC.

Keywords: Quantitative evaluation; histological grade; hepatocellular carcinoma (HCC); magnetic resonance image (MRI)

Submitted Mar 04, 2020. Accepted for publication May 14, 2020.

doi: $10.21037 /$ atm-20-2874

View this article at: http://dx.doi.org/10.21037/atm-20-2874 


\section{Introduction}

Despite improvements in diagnosis and patient management, hepatocellular carcinoma (HCC) was the sixth most commonly diagnosed cancer and the fourth leading cause of cancer death worldwide in 2018, especially in high risk areas like Asia and East Africa (1). The development of HCC may arise from de novo hepatocarcinogenesis or by a multistep progression from a regenerative nodule, to a dysplastic nodule, to small HCC (2). Approximately 90\% of HCCs are associated with a known underlying aetiology, such as chronic viral hepatitis (B and $\mathrm{C}$ ), additionally liver cirrhosis, which may be caused by chronic viral hepatitis, chronic alcohol abuse acquired and inherited metabolic diseases, is thought to be an important risk factor for HCC (3). Patients with cirrhosis, chronic hepatitis B viral infection, current or prior HCC are designated as those at high risk for HCC (4). As imaging technology advanced, in such high-risk patients, HCC can be diagnosed by typical imaging findings ("wash in" and "wash out") without pathological confirmation (5), even for HCC smaller than 3 centimeters.

The importance of early detection of HCC in at-risk populations has been emphasized, and advances in curative therapies, including surgical and local treatment, have contributed to the improved prognosis of patients with early stage HCC $(6,7)$. It has been demonstrated that tumor size is a risk factor for poor survival after resection of HCC, small HCC may have better outcome $(8,9)$. However, there are other pathological characteristics known as prognostic factors even in small HCCs, such as microvascular invasion or histological grade (10). Poor histological grades have been shown to correlate with unfavorable patient survival after liver transplantation, curative resection, and local therapies (11-14). Preoperative percutaneous biopsy is sometimes necessary for the diagnosis of hepatocellular carcinoma and its histological grading. However, invasive procedures may involve bleeding, sampling errors, and the risk of tumor seeding (15). Therefore, it is necessary to explore non-invasive diagnostic methods with effective predictive capabilities for the histological grade of HCC.

Many studies have contributed to the prediction of a poor prognosis of HCC by investigating preoperative imaging tools (16-18). Diffusion-weighted (DW) MR images have been shown as an effective tool for predicting histological grade (19-22). Previous studies demonstrated that some quantitative parameters such as lesion-to-liver contrast-to-noise ratio (CNR) or relative contrast ratio
(RCR) on gadoxetic acid (Gd-EOB-DTPA) enhanced T1weighted (T1W) imaging are feasible for predicting HCC grade (15,23-25). Compared with small HCCs, necrotic or cystic changes are more often seen in large HCCs, which may result in difficulties in avoiding necrotic or cystic component when placing the region of interest (ROI) of the tumor for quantitative analysis, and may contribute to measurement errors. There are few studies showing the correlation between RCR on T2W, gadobenate dimeglumine (Gd-BOPTA, another MRI contrast agent) enhanced T1W images including the hepatobiliary phase, and histological grade of small HCC. Therefore, focusing on small HCCs, this study aims to determine the predictive power of diffusion-weighted imaging (DWI), T2W, and dynamic Gd-BOPTA-enhanced T1W images for histological grade by using quantitative assessments.

\section{Methods}

This study was approved by the institutional review board of our institution. The requirement for written informed consent was waived for this retrospective study.

\section{Study population and design}

Medical records from September 2014 to June 2019 were reviewed to identify patients with surgically resected HCC who underwent a preoperative liver MRI examination with Gd-BOPTA in our hospital. Initially, 58 patients meeting the following eligibility criteria were included: (I) history of undergoing both pre-contrast and Gd-BOPTA-enhanced MRI examinations including the hepatobiliary phase (HBP) within a 4-week interval prior to surgery, (II) resected and pathologically confirmed HCC, (III) a tumor size smaller than or equal to $3 \mathrm{~cm}$, and (IV) no evidence of any previous HCC treatment. Subsequently, 9 patients were excluded due to the following exclusion criteria: (I) suboptimal imaging quality for interpretation $(\mathrm{n}=6)$ and (II) HCCs smaller than $6 \mathrm{~mm}$ in size excluded to avoid measurement bias due to partial volume effects $(n=3)$. Finally, 49 patients were recruited into this retrospective cohort. Our flowchart of inclusion and exclusion criteria for study patients is shown in Figure 1.

In addition, the medical records of all patients at hospital discharge were retrospectively reviewed for demographic data prior to MR examination within 1 week of surgery. Data included clinical symptoms, cause of disease, albumin level (grams per liter), total bilirubin level (milligram per 


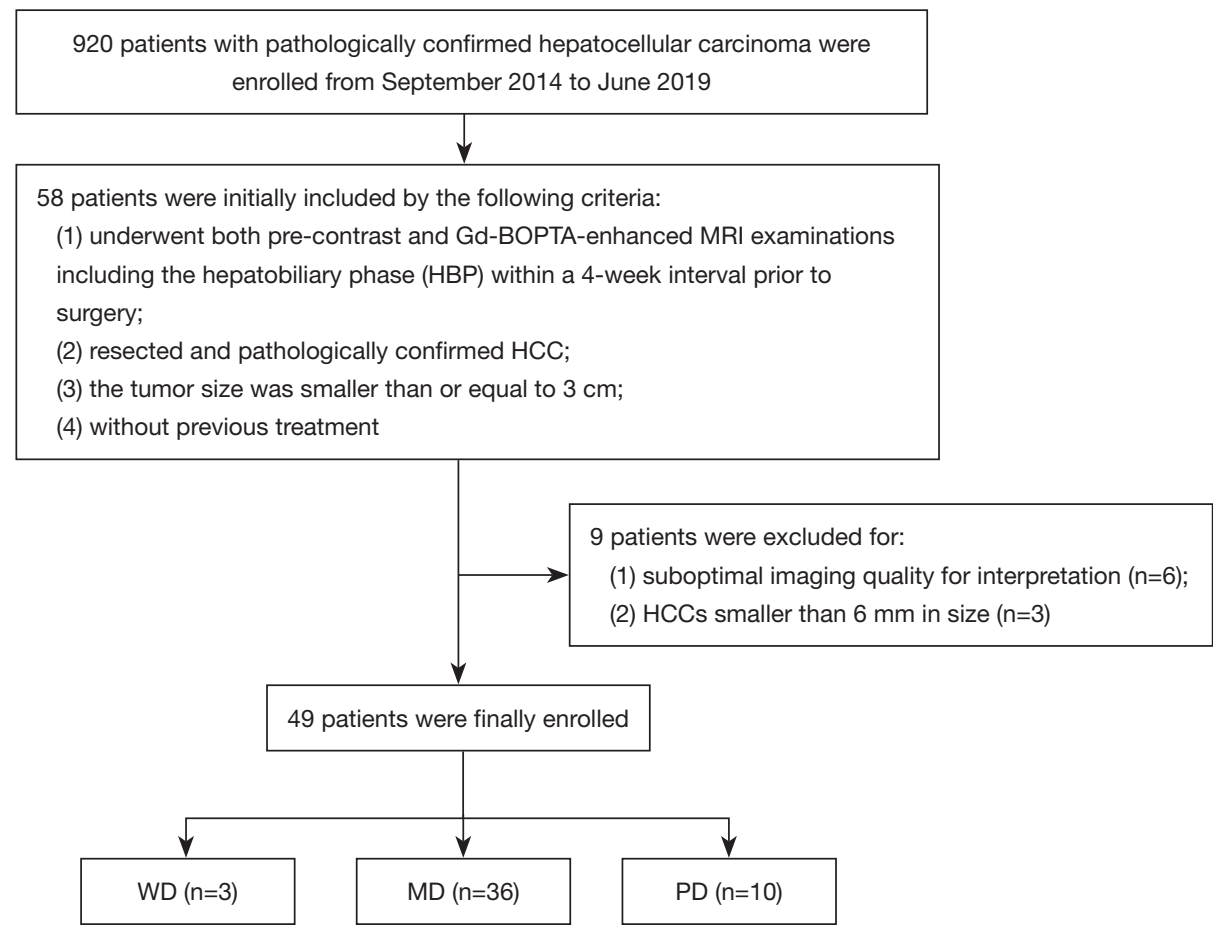

Figure 1 Flowchart of inclusion and exclusion criteria of study patients. Gd-BOPTA, gadobenate dimeglumine; MRI, magnetic resonance imaging; HCC, hepatocellular carcinoma; WD, well-differentiated; MD, moderately differentiated; PD, poorly differentiated.

deciliter), aspartate aminotransferase level (international unit per liter), alanine aminotransferase level (international unit per liter), and pre-operative serum alpha fetoprotein (AFP) levels (microgram per liter).

\section{MRI protocol}

A total of 49 cases were examined with 3.0T MRI system (Magnetom Verio; Siemens Healthcare, Erlangen, Germany) with an 8-channel body phased-array coil. Precontrast images were obtained using respiration-triggered $\mathrm{T} 2 \mathrm{~W}$ fat-suppression turbo spin-echo sequence [repetition time (TR), 6,500-7,000 ms; echo time (TE), $79 \mathrm{~ms}$; slice thickness, $5 \mathrm{~mm}$; matrix, 320×224; bandwidth, $240 \mathrm{~Hz} /$ pixel; gap, $1 \mathrm{~mm}$ ], a breath-hold T1-weighted gradient-echo inphase sequence (TR, $133 \mathrm{~ms}$; TE, $2.5 \mathrm{~ms}$; matrix, 320×224; bandwidth, $280 \mathrm{~Hz} /$ pixel), an out-of phase sequence (TR, 133 ms; TE, $1.2 \mathrm{~ms}$; matrix, 320×224; bandwidth, $280 \mathrm{~Hz} /$ pixel), a diffusion-weighted imaging (DWI) (TR, $6,300 \mathrm{~ms}$; TE, $93 \mathrm{~ms}$; gradient factor b values of 50 and $800 \mathrm{~s} / \mathrm{mm}^{2}$; matrix, $128 \times 128$; slice thickness, $3 \mathrm{~mm}$ ), and a T1-weighted three-dimensional (3D) volumetric interpolated breath-hold examination gradient-echo sequence with fat suppression (TR, $3.9 \mathrm{~ms}$; TE, $1.4 \mathrm{~ms}$; matrix, 320×224; bandwidth, $400 \mathrm{~Hz} /$ pixel; slice thickness, $3 \mathrm{~mm}$; gap, $0 \mathrm{~mm}$ ). For dynamic MRI, Gd-BOPTA (Bracco, Italy; $0.1 \mathrm{mmol} / \mathrm{kg}$ ) was injected as a rapid bolus and immediately followed by a $20 \mathrm{~mL}$ saline flush through a power injector at a rate of $2 \mathrm{~mL} / \mathrm{s}$. Dynamic imaging, including the arterial phase (AP) (20-25 s), portal venous phase (60-70 s), equilibrium phase ( $2 \mathrm{~min})$, and the delayed HBP $(60-120 \mathrm{~m})$, was performed using a T1-weighted 3D volumetric interpolated breath-hold examination with the equivalent parameters.

\section{Qualitative and quantitative imaging analyses}

All images were transferred to the Picture Archiving and Communication System (PACS). The following imaging parameters were evaluated for qualitative analysis: (I) enhancement pattern (arterial enhancement with washout on portal phase, arterial and persistent enhancement, and gradual enhancement); (II) internal necrotic or cystic portion, defined as bright-SI foci on T2W images without contrast enhancement; (III) intratumoral fat, defined as tumor area with decreased SI on opposed-phase images 
compared with in-phase images; (IV) hemorrhage, defined as high-SI foci on T1-weighted images with variable SI on T2W images; and $(\mathrm{V})$ tumor capsule, defined as a distinct low-SI rim on arterial phase images with delayed contrast enhancement surrounding a tumor margin involving more than $90 \%$ of the tumor circumference (18).

Quantitative image analysis of DWI, T2WI, and nonenhanced and contrast-enhanced T1WI were independently analyzed by 2 experienced radiologists blinded to the histopathological results. These specialists had over 5 and 10 years of experience in liver imaging. They placed the ROIs of each tumor independently, and manually adjusted it to ensure that the tumor was located in roughly the same position in different sequences. The SI of the tumor was measured using a ROI on T2W, DWI, dynamic, and hepatobiliary phase Gd-BOPTA-enhanced T1W imaging for quantitative analysis. To avoid partial volume calculation errors, the ROI of each tumor was placed at the slice where the tumor was best visualized, and the images were chosen which were closest to the largest diameter of the tumor. In case of heterogeneous enhancement, the ROI was placed at the part corresponding to the wash out of each tumor. To exclude the influence of the environment at the time of the examination and the composition of the patient, the SI of the tumors was corrected by the SI of the paraspinous muscles, which were also measured in the ROI in the same slice. The ROI was chosen to deliberately avoid necrotic and cystic degeneration, and hemorrhagic blood vessels areas. Areas selected were round or oval shaped and approximately 0.5 to $1.0 \mathrm{~cm}^{2}$, with the average of the 3 measurements represented. We calculated the parameters for T2WI, DWI, dynamic, and hepatobiliary phase Gd-BOPTA-enhanced T1W imaging according to the following equation: RCR $=$ SI (lesion) $/$ SI (paraspinous muscles).

\section{Histopathological evaluation}

All surgical liver specimens were obtained within 4 weeks of Gd-BOPTA-enhanced MRI. Specimens were fixed in 10\% neutral-buffered formalin, subjected to alcohol dehydration, xylene transparency, and paraffin embedding. These lesions were processed for histological examination (hematoxylin and eosin staining). Gross and microscopic analyses of all specimens were performed by an experienced pathologist. Histologic HCC grading was performed using the modified version of the Edmonson and Steiner classification (26,27). Specifically, modified Edmondson and Steiner grade 1 was defined as well-differentiated (WD), grade 2 as moderately differentiated (MD), and grade 3 as poorly differentiated (PD). Moreover, the final grade of HCC was established according to the highest grade assessed. Ultimately, the 49 small HCCs consisted of 3 WD HCCs, 36 MD HCCs, and 10 PD HCCs.

\section{Statistical analysis}

The data were analyzed with SPSS software 18.0 (IBMSPSS, Chicago, IL, USA). Means (standard deviations), medians (ranges), or frequencies were used to describe patient baseline characteristics. The reproducibility of the RCR of small HCC was assessed by determination of the intraclass correlation coefficient (ICC), which reflected the differences in reliability between the 2 independent radiologists. ICC values less than 0.5 were indicative of poor reliability, values between 0.5 and 0.75 indicated moderate reliability, values between 0.75 and 0.9 indicated good reliability, and values greater than 0.90 indicated excellent reliability. Chi square test or Fisher's exact test was used to analyze the correlation between categorical variables and histological grades. The Spearman's rank correlation test was used to analyze the correlation between histological grade and T2WI RCR, DWI RCR, precontrast T1WI RCR, arterial phase RCR, portal phase RCR, equilibrium phase RCR, and hepatobiliary phase RCR. The degree of correlation was defined by the absolute value of the correlation coefficient $(\rho)$ as follows: strong, 0.70 or greater; moderate, 0.40 to 0.70 ; weak, 0.2 to 0.4 ; or none, less than 0.2 . A positive $\rho$ value was defined as a direct correlation, and a negative $\rho$ value was defined as a negative correlation. $\mathrm{P}$ values less than 0.05 were considered to be statistically significant.

\section{Results}

\section{Baseline characteristics of patients}

Of the 49 patients, 39 were men and 10 were women. In terms of location, $61 \%(30 / 49)$ of the lesions were located in the right lobe, and $39 \%(19 / 49)$ were in the left lobe. Overall, these lesions were classified as 3 WD HCCs, 36 MD HCCs, and 10 PD HCCs. Furthermore, 45 patients out of the total study population experienced type $B$ viral hepatitis while the remaining 4 experienced no history of type B or C viral hepatitis. The severity of liver malfunction was evaluated according to the Child-Pugh classification resulting in 47 patients classified as grade A 
and 2 patients as grade B. Serum AFP was greater than normal values $(0-25 \mu \mathrm{g} / \mathrm{L})$ in 30 patients, lower than the normal levels in 16 patients, and not available for 3 patients prior to MR examinations (Table 1). All 49 small HCCs showed high SI on DWI, and all lesions had slightly high SI on T2WI. Regarding the pattern types, 31 small HCCs displayed typical contrast patterns (arterial enhancement with washout on portal phase) (WD/MD/PD: 1/28/2, respectively), and 18 had atypical contrast patterns (arterial enhancement with persistent enhancement on portal phase

Table 1 Patient and tumor characteristics

\begin{tabular}{lc}
\hline Characteristic & Value \\
\hline Median age [range], y & 52 [23-85] \\
Median size (range), cm & $2.10(0.76-3.0)$ \\
Sex (male/female) & $39 / 10$ \\
Child-Pugh classification (A/B/C) & $47 / 2 / 0$ \\
Median T-Bil level (range), mg/mL & $32(11.6-42.2)$ \\
Liver cirrhosis, presence, $\mathrm{n}(\%)$ & $32(72.7)$ \\
Median AFP (range), ng/mL & $53.8(1.59-2,000)$ \\
Tumor location & 30 \\
Right & 19 \\
Left & $2.1(0.8-3.0)$ \\
Median tumor diameter (range), cm & $(3 / 36 / 10)$ \\
Histological tumor grade (well/moderate/poor) & \\
\hline AFP, alpha fetoprotein; Well, well-differentiated; Moderate, \\
moderately differentiated; Poor, poorly differentiated.
\end{tabular}

or gradual enhancement on portal phase) (WD/MD/PD: $2 / 8 / 8$, respectively).

As shown in Table 2, All ICC values between 0.5 and 0.90 indicated moderate to good reliability. The relationships between the small HCC histological grade and each variable are shown in Figure 2 and Table 3, there was no correlation between tumor margin, tumor location, cystic/necrotic change, intratumoral fat, enhancement pattern, tumor capsule, tumor boundary, or tumor size among any of the differentiation categories ( $\mathrm{P}>0.05$ for all).

There was a statistically significant correlation between RCR and small HCC in arterial phase images $(\rho=-0.305$, $\mathrm{P}<0.05)$. In terms of multiple comparisons, the RCR of the small PD HCCs was significantly lower than that of the small WD HCCs $(\mathrm{P}<0.05)$, whereas no significant difference existed between small WD HCCs and MD HCCs ( $\mathrm{P}=0.083)$ (Figures 3,4), or between small MD HCCs and PD HCCs $(\mathrm{P}=0.076)$.

Additionally, there were no statistically significant differences between the age, tumor size, and RCR in other phases among the different differentiation classifications of small HCCs.

As detailed in Table 4, there was no significant correlation between tumor margin, tumor location, cystic/necrotic change, steatosis, enhancement pattern, tumor capsule, tumor boundary, or tumor size across the differentiated categories of small HCC groups $(\mathrm{P}>0.05$ for all).

\section{Discussion}

The staging of HCC is challenging not only due to the complexity of background factors such as liver function

Table 2 Comparison of RCR measurements

\begin{tabular}{|c|c|c|c|c|}
\hline Parameter & Reviewer 1 & Reviewer 2 & ICC & $\mathrm{P}$ \\
\hline DWI RCR & $1.63 \pm 0.70$ & $1.75 \pm 0.78$ & 0.628 & $<0.001$ \\
\hline Precontrast T1WI RCR & $0.98 \pm 0.20$ & $0.99 \pm 0.21$ & 0.576 & $<0.001$ \\
\hline Arterial phase RCR & $1.90 \pm 0.73$ & $1.96 \pm 0.57$ & 0.869 & $<0.001$ \\
\hline Equilibrium phase RCR & $1.54 \pm 0.43$ & $1.54 \pm 0.38$ & 0.802 & $<0.001$ \\
\hline Hepatobiliary phase RCR & $1.27 \pm 0.26$ & $1.26 \pm 0.24$ & 0.813 & $<0.001$ \\
\hline
\end{tabular}

Data are presented as mean $\pm \mathrm{SD}$, unless otherwise indicated. $\mathrm{RCR}$, relative contrast ratio. 
and history of liver cirrhosis, but also due to the size of the tumor, lymph infiltration, metastasis, and the evaluation of tumor invasiveness according to its histological features (28). Even though as an important indicator to represent the aggressiveness of the tumor, histological grading of HCC is not routinely given by tissue biopsy before surgical intervention because of the invasiveness and concern

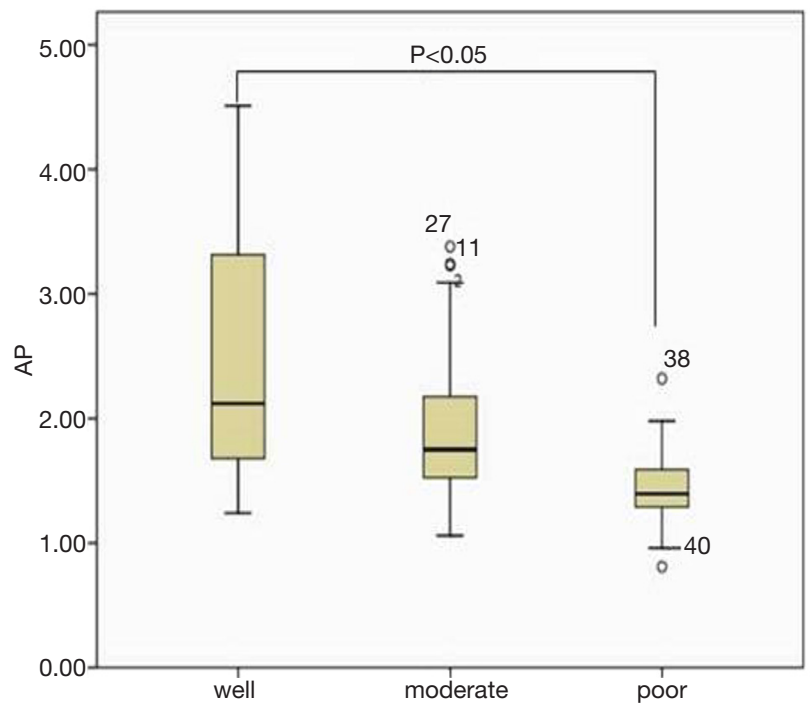

Figure 2 The relationship between the small HCC histological grade and each variable on the arterial phase images. Boxplots of the AP RCR of well-differentiated (well), moderately differentiated (moderate), and poorly differentiated (poor) HCCs are shown. HCC, hepatocellular carcinoma; AP, arterial phase; RCR, relative contrast ratio. for procedure-related complications. Many years of research and verification have outlined the potential use of quantitative assessments for depicting the histopathologic grade of HCC. Several studies have additionally shown that qualitative and quantitative examination of the Gd-BOPTA image is of great value in the differential diagnosis of focal liver lesions (29). However, Gd-BOPTA-enhanced MRI has rarely been used to evaluate histological grades of HCC (30-32), and even less so in the evaluation of small HCC.

In this study, the contrast agent used was gadobenate dimeglumine (Gd-BOPTA) which was commonly used in previous reports $(15,25,33)$. In contrast to previous studies, where the liver parenchyma surrounding the lesion was usually selected as the reference tissue, we corrected the SI of tumors using the spinal upright muscle as an internal reference, as proposed in previous studies $(34,35)$. The rationale is that the parenchyma may be affected by liver and renal function, obstructing the accuracy of measurements within the region of interest (ROI) which may be affected by adjacent blood vessels and bile ducts (36). Selecting the spinal upright muscle eliminates those potential confounds.

This study demonstrated that the correlation between RCR and small HCC in arterial phase images is statistically significant. There was a significant difference in the RCR between well- and PD HCCs, though not among other histological grade combinations. In previous studies regarding intravoxel incoherent motion, the perfusion fraction has been shown to be positively correlated with the contrast agent enhancement ratio in the arterial phase (30).

Table 3 Comparisons of different parameters among different histological grades of small HCCs

\begin{tabular}{|c|c|c|c|c|}
\hline Parameters & WD HCC & MD HCC & PD HCC & $\mathrm{P}$ \\
\hline Size, cm & $1.92 \pm 0.83$ & $2.03 \pm 0.57$ & $1.97 \pm 0.90$ & 0.942 \\
\hline T2WI RCR & $3.77 \pm 2.90$ & $2.62 \pm 0.86$ & $2.42 \pm 0.61$ & 0.128 \\
\hline DWI RCR & $1.15 \pm 0.56$ & $1.65 \pm 0.66$ & $1.68 \pm 0.78$ & 0.474 \\
\hline Arterial phase RCR & $2.62 \pm 1.69$ & $1.90 \pm 0.62$ & $1.46 \pm 0.44$ & 0.033 \\
\hline Portal phase RCR & $2.04 \pm 1.18$ & $1.66 \pm 0.54$ & $1.48 \pm 0.36$ & 0.313 \\
\hline Equilibrium phase RCR & $2.02 \pm 0.91$ & $1.47 \pm 0.42$ & $1.41 \pm 0.25$ & 0.088 \\
\hline Hepatobiliary phase RCR & $1.43 \pm 0.27$ & $1.22 \pm 0.31$ & $1.20 \pm 0.19$ & 0.453 \\
\hline
\end{tabular}

Data in parentheses are percentages. HCC, hepatocellular carcinoma; WD, well-differentiated; MD, moderately differentiated; PD, poorly differentiated; DWI, diffusion-weighted imaging; RCR, relative contrast ratio. 

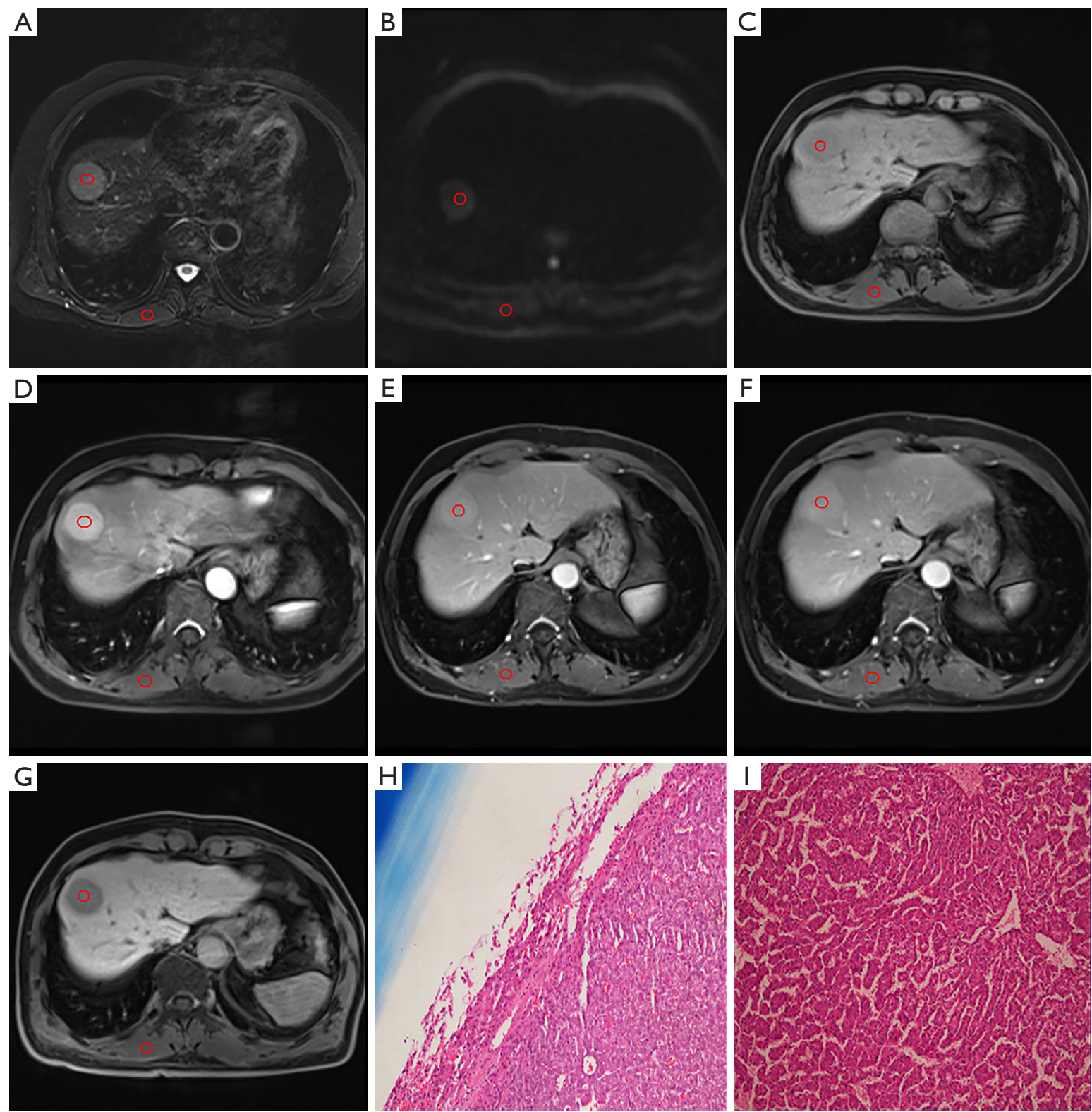

Figure 3 A patient with well-differentiated small HCC with MR images displaying the placement of ROIs for the measurement of signal intensities. The RCR of T2WI (A), DWI (B), pre-contrast T1WI (C), arterial phase (D), portal phase (E), equilibrium phase (F), and hepatobiliary phase $(\mathrm{G})$ were $2.09,0.87,1.38,2.08,1.30,1.75$, and 1.36, respectively. (H,I) showing well-differentiated small HCC. H\&E staining results ( $\times 400$ magnification). HCC, hepatocellular carcinoma; MR, magnetic resonance; RCR, relative contrast ratio; DWI, diffusion-weighted imaging.

In our study, the mean arterial phase RCR of WD HCCs tended to be higher than that of other histological grades.

Other images such as DWI RCR could not predict the pathological stage of small HCCs. Many previous studies have evaluated ADC measurements to predict histological grading of HCC, although with different results. One of the reasons for this discrepancy may be rooted in the inherent problem in computing ADC in the first place (19-22,37,38). Ultimately, a variety of factors affect the reliability of $\mathrm{ADC}$ calculations, such as respiratory movement, cardiac movement, and partial volume effects, especially for smaller HCCs. Although previous studies have shown that the 

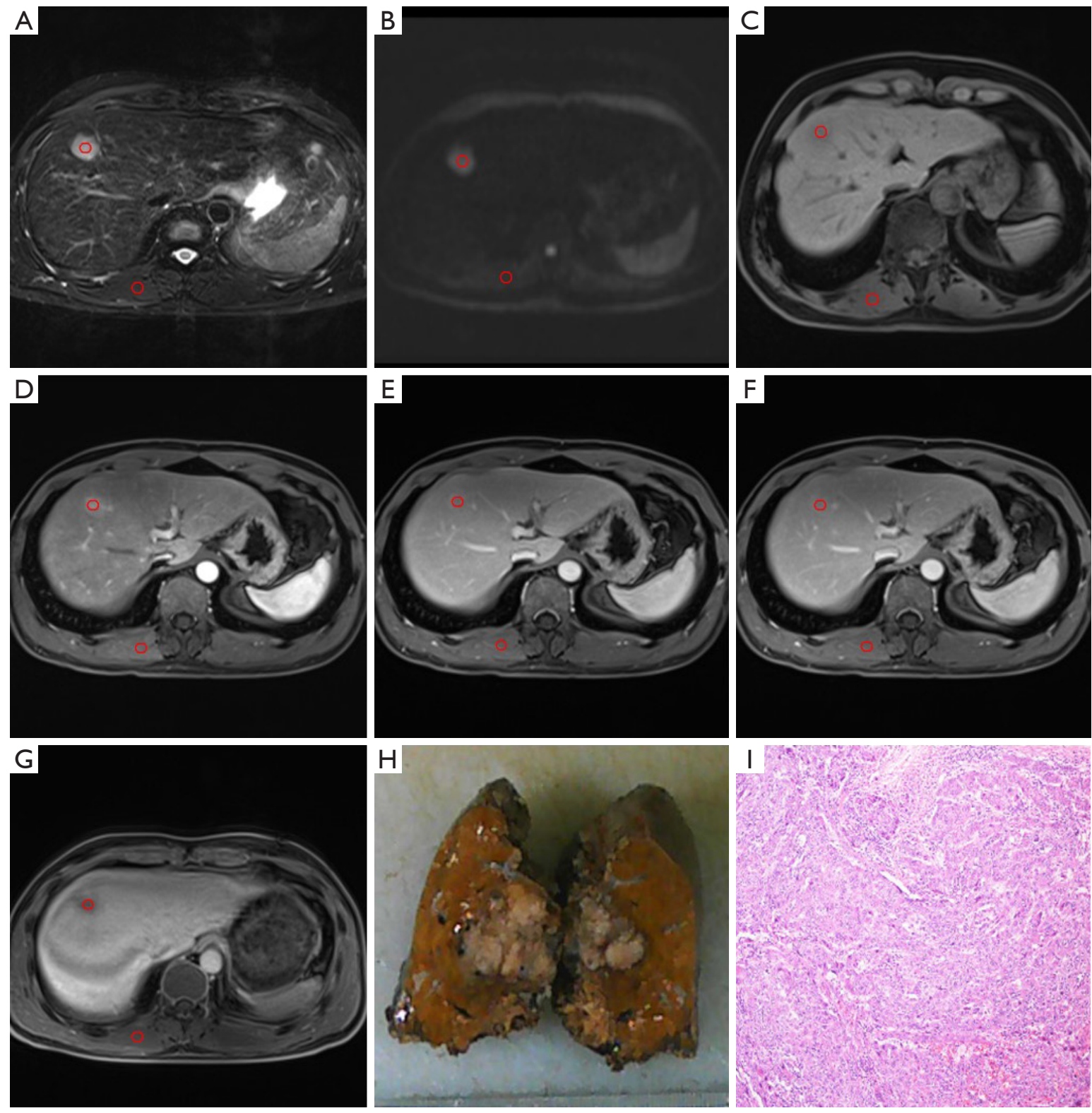

Figure 4 A patient with moderately differentiated small HCC of MR images in the T2WI, DWI, dynamic phase-enhanced T1W imaging to display the placement of ROIs for the measurement of signal intensities. The RCR of T2WI (A), DWI (B), pre-contrast T1WI (C), arterial phase (D), portal phase (E), equilibrium phase (F), and hepatobiliary phase (G) were 3.23, 1.86, 0.79, 1.36, 0.98, 1.01, and 1.14 respectively. (H,I) showing moderately differentiated small HCC. H\&E staining results (×400 magnification). HCC, hepatocellular carcinoma; MR, magnetic resonance; DWI, diffusion-weighted imaging; RCR, relative contrast ratio.

reproducibility of DWI RCR is superior to that of ADC, it can be used as an alternative method for predicting histological grading of HCC (33).

We found no statistical differences between tumor size and RCR in other phases among the differently differentiated small HCCs. However, previous literature has shown the RCR on DWI, T2WI, or HBP images can predict the pathological stage of HCC (33). Differences between our and their findings may be due to the different reference points used or perhaps their sole focus on HCC as a whole as opposed to our categorical approach.

Several limitations of the present study should be 
Table 4 Comparisons of different imaging features among 3 histological grades of small HCCs

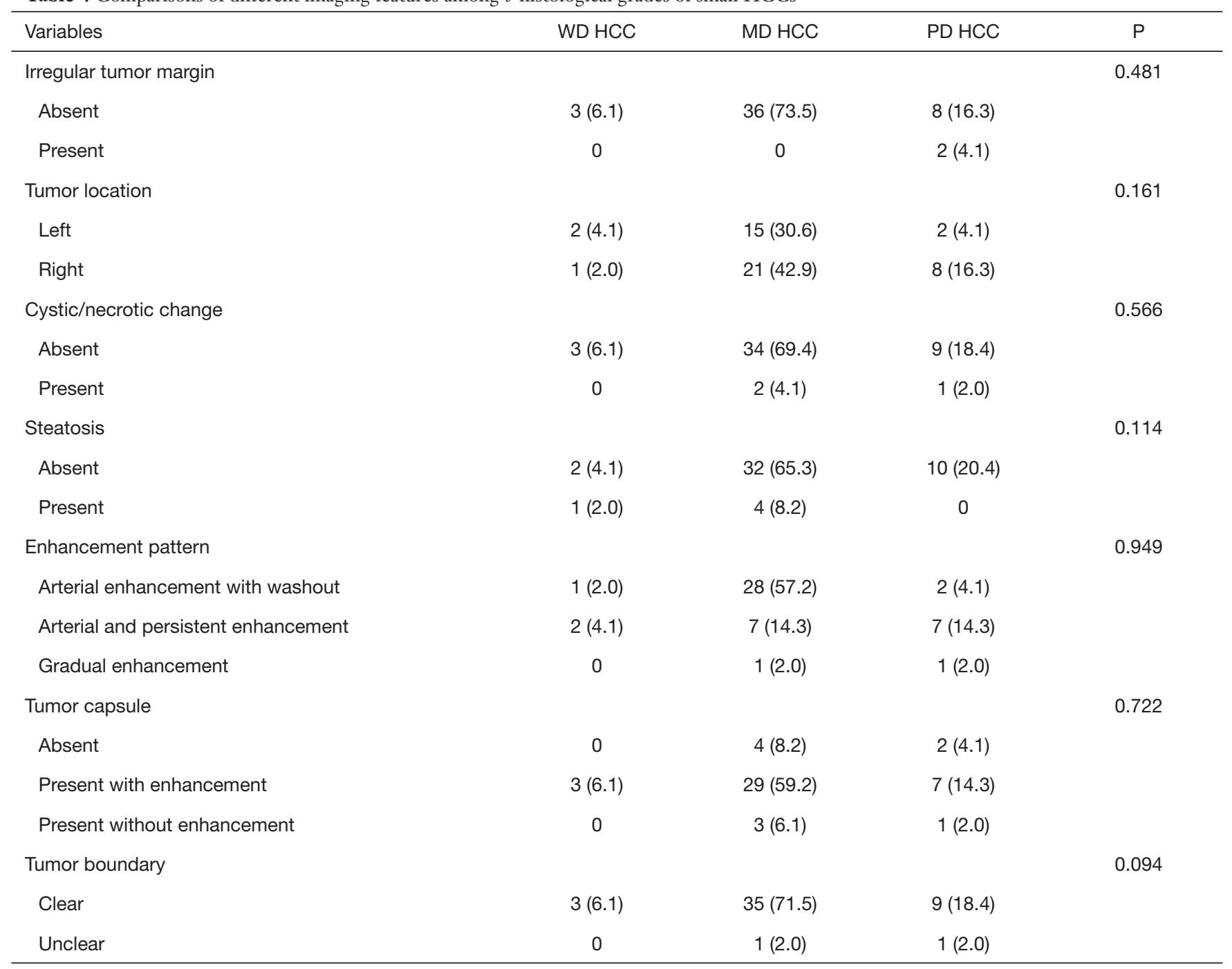

Data are numbers (percentage). HCC, hepatocellular carcinoma; WD, well-differentiated; MD, moderately differentiated; PD, poorly differentiated.

mentioned. First, the number of liver cancer lesions included in this study were relatively small. Second, because we enrolled only cases which had undergone hepatic resection, the number of WD HCCs represented were even smaller. As such, these may be insufficient for assessing statistical significance for the histological grade category. Finally, this study was conducted at a single facility, possibly introducing a selection bias in our patient population. However, the aim of this study was to identify the diagnostic performance of quantitative MRI parameters in identifying 3 histological levels of small HCCs. All HCCs in this study were confirmed by histopathological examination of hepatectomized specimens. Therefore, the probability of sampling error is low.

In this study, we found that preoperative quantitative MRI parameters may be useful tools for predicting the histological grade of small HCC. This methodology is less invasive than current biopsy procedures and offers important characteristics that can help guide the treatment plan. Specifically, relatively low arterial enhancement on gadobenate dimeglumine MR images is demonstrated herein as a predictor of deteriorating histological grades of small HCCs.

\section{Conclusions}

In conclusion, the present study indicates that the lesion- 
to-paraspinous muscle RCR on arterial phase Gd-BOPTAenhanced T1W imaging may be useful for the prediction of the histological characteristics of small HCC. Such quantitative radiomics models of small HCC may potentially be useful for precision medicine and affect patient treatment strategies.

\section{Acknowledgments}

We thank the radiographers at the First Affiliated Hospital of Fujian Medical University for scanning the patients and data collections in this study.

Funding: This study received funding from the Joint Funds for the Innovation of Science and Technology, Fujian province (CN) (Award Number: 2019Y9125).

\section{Footnote}

Data Sharing Statement: Available at http://dx.doi. org/10.21037/atm-20-2874

Conflicts of Interest: All authors have completed the ICMJE uniform disclosure form (available at http://dx.doi. org/10.21037/atm-20-2874). The authors have no conflicts of interest to declare.

Ethical Statements: The authors are accountable for all aspects of the work in ensuring that questions related to the accuracy or integrity of any part of the work are appropriately investigated and resolved. The study was conducted in accordance with the Declaration of Helsinki (as revised in 2013). This single-center retrospective cohort study was approved by the Institutional Review Board \{no. [2016]118 and no. [2019]283\}, who determined the requirement for informed consent could be waived.

Open Access Statement: This is an Open Access article distributed in accordance with the Creative Commons Attribution-NonCommercial-NoDerivs 4.0 International License (CC BY-NC-ND 4.0), which permits the noncommercial replication and distribution of the article with the strict proviso that no changes or edits are made and the original work is properly cited (including links to both the formal publication through the relevant DOI and the license). See: https://creativecommons.org/licenses/by-nc-nd/4.0/.

\section{References}

1. Bray F, Ferlay J, Soerjomataram I, et al. Global cancer statistics 2018: GLOBOCAN estimates of incidence and mortality worldwide for 36 cancers in 185 countries. CA Cancer J Clin 2018;68:394-424.

2. Holzwanger DJ, Madoff DC. Role of interventional radiology in the management of hepatocellular carcinoma: current status. Chin Clin Oncol 2018;7:49.

3. European Association for the Study of the Liver. Electronic address eee, European Association for the Study of the L. EASL Clinical Practice Guidelines: Management of hepatocellular carcinoma. J Hepatol 2018;69:182-236.

4. Chernyak V, Fowler KJ, Kamaya A, et al. Liver Imaging Reporting and Data System (LI-RADS) Version 2018: Imaging of Hepatocellular Carcinoma in At-Risk Patients. Radiology 2018;289:816-30.

5. Marrero JA, Kulik LM, Sirlin CB, et al. Diagnosis, Staging, and Management of Hepatocellular Carcinoma: 2018 Practice Guidance by the American Association for the Study of Liver Diseases. Hepatology 2018;68:723-50.

6. Bruix J, Llovet JM. Major achievements in hepatocellular carcinoma. Lancet 2009;373:614-6.

7. Mak LY, Cruz-Ramon V, Chinchilla-Lopez P, et al. Global Epidemiology, Prevention, and Management of Hepatocellular Carcinoma. Am Soc Clin Oncol Educ Book 2018;38:262-79.

8. Minagawa M, Ikai I, Matsuyama Y, et al. Staging of hepatocellular carcinoma: assessment of the Japanese TNM and AJCC/UICC TNM systems in a cohort of 13,772 patients in Japan. Ann Surg 2007;245:909-22.

9. Vauthey JN, Lauwers GY, Esnaola NF, et al. Simplified staging for hepatocellular carcinoma. J Clin Oncol 2002;20:1527-36.

10. Zhu Q, Yuan B, Qiao GL, et al. Prognostic factors for survival after hepatic resection of early hepatocellular carcinoma in HBV-related cirrhotic patients. Clin Res Hepatol Gastroenterol 2016;40:418-27.

11. Jonas S, Bechstein WO, Steinmuller T, et al. Vascular invasion and histopathologic grading determine outcome after liver transplantation for hepatocellular carcinoma in cirrhosis. Hepatology 2001;33:1080-6.

12. Bae SH, Park HC. Local modalities for inoperable hepatocellular carcinoma: radiofrequency ablation versus stereotactic body radiotherapy. Ann Transl Med 2018;6:S3.

13. Lauwers GY, Terris B, Balis UJ, et al. Prognostic histologic indicators of curatively resected hepatocellular carcinomas: a multi-institutional analysis of 425 patients with definition of a histologic prognostic index. Am J Surg Pathol 2002;26:25-34.

14. Oishi K, Itamoto T, Amano H, et al. Clinicopathologic 
features of poorly differentiated hepatocellular carcinoma. J Surg Oncol 2007;95:311-6.

15. Choi JY, Kim MJ, Park YN, et al. Gadoxetate disodiumenhanced hepatobiliary phase MRI of hepatocellular carcinoma: correlation with histological characteristics. AJR Am J Roentgenol 2011;197:399-405.

16. Li Y, Yan C, Weng S, et al. Texture analysis of multi-phase MRI images to detect expression of Ki67 in hepatocellular carcinoma. Clin Radiol 2019;74:813.e19-27.

17. Li Y, Chen J, Weng S, et al. Small hepatocellular carcinoma: using MRI to predict histological grade and Ki-67 expression. Clin Radiol 2019;74:653.e1-9.

18. Choi SY, Kim SH, Park CK, et al. Imaging Features of Gadoxetic Acid-enhanced and Diffusion-weighted MR Imaging for Identifying Cytokeratin 19-positive Hepatocellular Carcinoma: A Retrospective Observational Study. Radiology 2018;286:897-908.

19. Nakanishi M, Chuma M, Hige S, et al. Relationship between diffusion-weighted magnetic resonance imaging and histological tumor grading of hepatocellular carcinoma. Ann Surg Oncol 2012;19:1302-9.

20. Nishie A, Tajima T, Asayama Y, et al. Diagnostic performance of apparent diffusion coefficient for predicting histological grade of hepatocellular carcinoma. Eur J Radiol 2011;80:e29-33.

21. Chang WC, Chen RC, Chou CT, et al. Histological grade of hepatocellular carcinoma correlates with arterial enhancement on gadoxetic acid-enhanced and diffusionweighted MR images. Abdom Imaging 2014;39:1202-12.

22. Saito K, Moriyasu F, Sugimoto K, et al. Histological grade of differentiation of hepatocellular carcinoma: comparison of the efficacy of diffusion-weighted MRI with T2weighted imaging and angiography-assisted CT. J Med Imaging Radiat Oncol 2012;56:261-9.

23. Kogita S, Imai Y, Okada M, et al. Gd-EOB-DTPAenhanced magnetic resonance images of hepatocellular carcinoma: correlation with histological grading and portal blood flow. Eur Radiol 2010;20:2405-413.

24. Li Y, Lin D, Weng Y, et al. Early Diffusion-Weighted Imaging and Proton Magnetic Resonance Spectroscopy Features of Liver Transplanted Tumors Treated with Radiation in Rabbits: Correlation with Histopathology. Radiat Res 2019;191:52-9.

25. Jin YJ, Cho SG, Lee KY, et al. Association between relative liver enhancement on gadoxetic acid enhanced magnetic resonance images and histologic grade of hepatocellular carcinoma. Medicine (Baltimore) 2017;96:e7580.

26. Edmondson HA, Steiner PE. Primary carcinoma of the liver: a study of 100 cases among 48,900 necropsies. Cancer 1954;7:462-503.

27. Nzeako UC, Goodman ZD, Ishak KG. Comparison of tumor pathology with duration of survival of North American patients with hepatocellular carcinoma. Cancer 1995;76:579-88.

28. Willatt JM, Hussain HK, Adusumilli S, et al. MR Imaging of hepatocellular carcinoma in the cirrhotic liver: challenges and controversies. Radiology 2008;247:311-30.

29. Kim TK, Lee KH, Jang HJ, et al. Analysis of gadobenate dimeglumine-enhanced MR findings for characterizing small $(1-2-\mathrm{cm})$ hepatic nodules in patients at high risk for hepatocellular carcinoma. Radiology 2011;259:730-8.

30. Grazioli L, Morana G, Caudana R, et al. Hepatocellular carcinoma: correlation between gadobenate dimeglumineenhanced MRI and pathologic findings. Invest Radiol 2000;35:25-34.

31. Vogl TJ, Stupavsky A, Pegios W, et al. Hepatocellular carcinoma: evaluation with dynamic and static gadobenate dimeglumine-enhanced MR imaging and histopathologic correlation. Radiology 1997;205:721-8.

32. Tahir B, Sandrasegaran K, Ramaswamy R, et al. Does the hepatocellular phase of gadobenate dimeglumine help to differentiate hepatocellular carcinoma in cirrhotic patients according to histological grade? Clin Radiol 2011;66:845-52.

33. Iwasa Y, Kitazume Y, Tateishi U, et al. Hepatocellular Carcinoma Histological Grade Prediction: A Quantitative Comparison of Diffusion-Weighted, T2-Weighted, and Hepatobiliary-Phase Magnetic Resonance Imaging. J Comput Assist Tomogr 2016;40:463-70.

34. Huang X, Xiao Z, Zhang Y, et al. Hepatocellular Carcinoma: Retrospective Evaluation of the Correlation Between Gadobenate Dimeglumine-Enhanced Magnetic Resonance Imaging and Pathologic Grade. J Comput Assist Tomogr 2018;42:365-72.

35. Okamura S, Sumie S, Tonan T, et al. Diffusion-weighted magnetic resonance imaging predicts malignant potential in small hepatocellular carcinoma. Dig Liver Dis 2016;48:945-52.

36. Kim H, Kim MJ, Park MS, et al. Potential conditions causing impairment of selective hepatobiliary enhancement of gadobenate dimeglumine-enhanced delayed magnetic resonance imaging. J Comput Assist Tomogr 2010;34:113-20.

37. Muhi A, Ichikawa T, Motosugi U, et al. High-b-value diffusion-weighted MR imaging of hepatocellular lesions: estimation of grade of malignancy of hepatocellular 
carcinoma. J Magn Reson Imaging 2009;30:1005-11.

38. Nasu K, Kuroki Y, Tsukamoto T, et al. Diffusionweighted imaging of surgically resected hepatocellular carcinoma: imaging characteristics and relationship among signal intensity, apparent diffusion coefficient, and histopathologic grade. AJR Am J Roentgenol 2009;193:438-44.

Cite this article as: Weng S, Xu X, Li Y, Yan C, Chen J, Ye R, Zhu Y, Wen L, Hong J. Quantitative analysis of multiphase magnetic resonance images may assist prediction of histopathological grade of small hepatocellular carcinoma. Ann Transl Med 2020;8(16):1023. doi: 10.21037/atm-20-2874 